\title{
Atlas of the Global Burden of Stroke (1990-2013): The GBD 2013 Study
}

\author{
Valery L. Feigin ${ }^{a}$ George A. Mensah ${ }^{d}$ Bo Norrving ${ }^{b}$ Christopher J.L. Murray ${ }^{c}$ \\ Gregory A. Roth ${ }^{c}$ GBD 2013 Stroke Panel Experts Group \\ aFAAN, National Institute for Stroke and Applied Neurosciences, Auckland University of Technology, Auckland, \\ New Zealand; 'b Department of Clinical Sciences, Neurology, Lund University, Lund, Sweden; ' Institute for Health \\ Metrics and Evaluation and the Division of Cardiology, School of Medicine, University of Washington, Seattle, Wash., \\ ${ }^{\mathrm{d} C e n t e r}$ for Translation Research and Implementation Science and Division of Cardiovascular Sciences; National \\ Heart, Lung, and Blood Institute; National Institutes of Health, Bethesda, Md., USA
}

\section{Key Words}

Stroke · Atlas · Burden · GBD 2013

\section{Abstract}

Background: World mapping is an important tool to visualize stroke burden and its trends in various regions and countries. Objectives: To show geographic patterns of incidence, prevalence, mortality, disability-adjusted life years (DALYs) and years lived with disability (YLDs) and their trends for ischemic stroke and hemorrhagic stroke in the world for 1990-2013. Methodology: Stroke incidence, prevalence, mortality, DALYs and YLDs were estimated following the general approach of the Global Burden of Disease (GBD) 2010 with several important improvements in methods. Data were updated for mortality (through April 2014) and stroke incidence, prevalence, case fatality and severity through 2013. Death was estimated using an ensemble modeling approach. A new software package, DisMod-MR 2.0, was used as part of a custom modeling process to estimate YLDs. All rates were age-standardized to new GBD es- timates of global population. All estimates have been computed with 95\% uncertainty intervals. Results: Age-standardized incidence, mortality, prevalence and DALYs/YLDs declined over the period from 1990 to 2013 . However, the absolute number of people affected by stroke has substantially increased across all countries in the world over the same time period, suggesting that the global stroke burden continues to increase. There were significant geographical (country and regional) differences in stroke burden in the world, with the majority of the burden borne by low- and middle-income countries. Conclusions: Global burden of stroke has continued to increase in spite of dramatic declines in age-standardized incidence, prevalence, mortality rates and disability. Population growth and aging have played an important role in the observed increase in stroke burden.

(c) 2015 S. Karger AG, Basel

Members of the GBD 2013 Stroke Panel Experts Group are listed in the Appendix at the end of the paper.

\section{KARGER 125}

C 2015 S. Karger AG, Basel

$0251-5350 / 15 / 0453-0230 \$ 39.50 / 0$ 


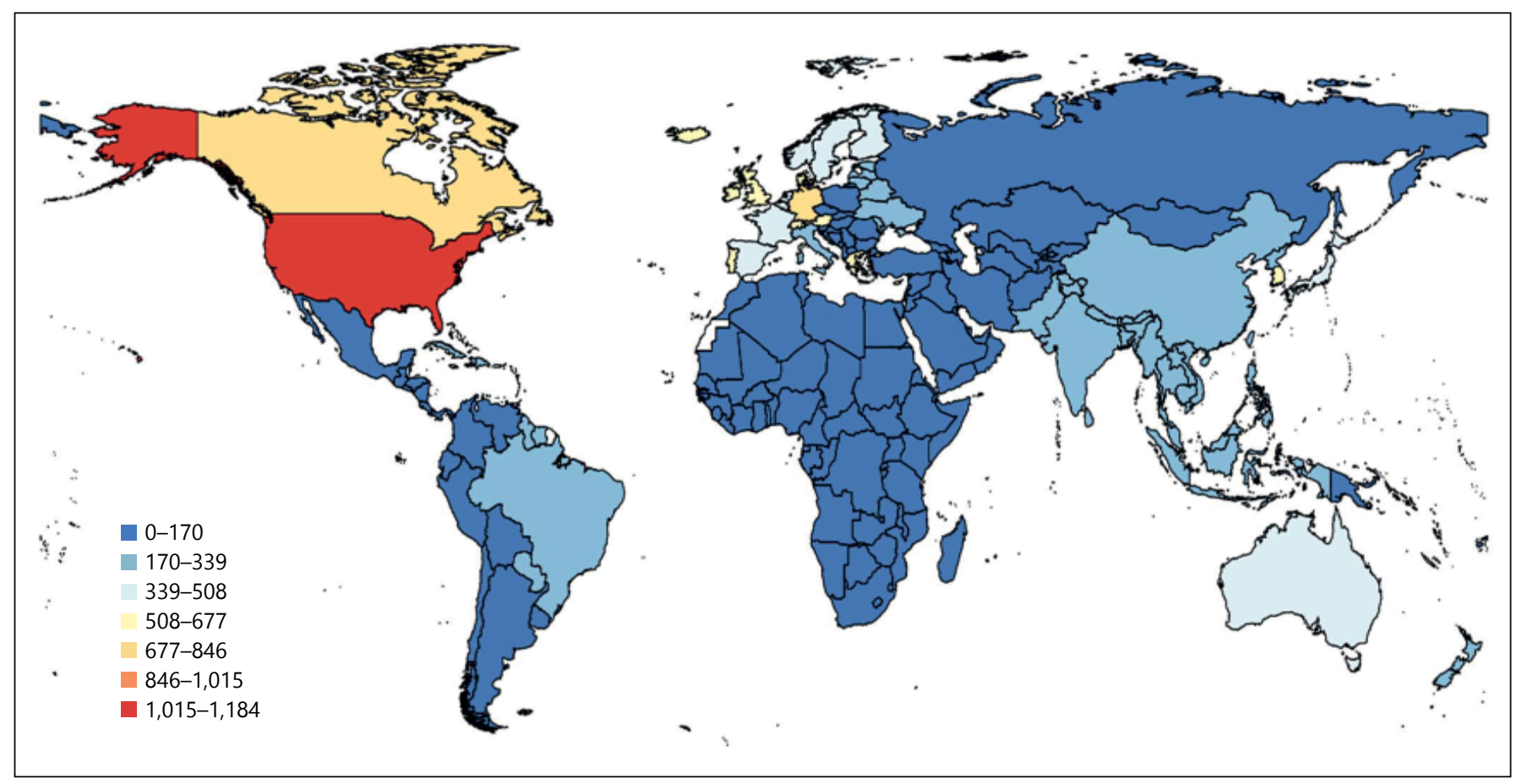

Fig. 1. Age-standardized annual prevalence (per 100,000) of ischemic stroke in 2013.

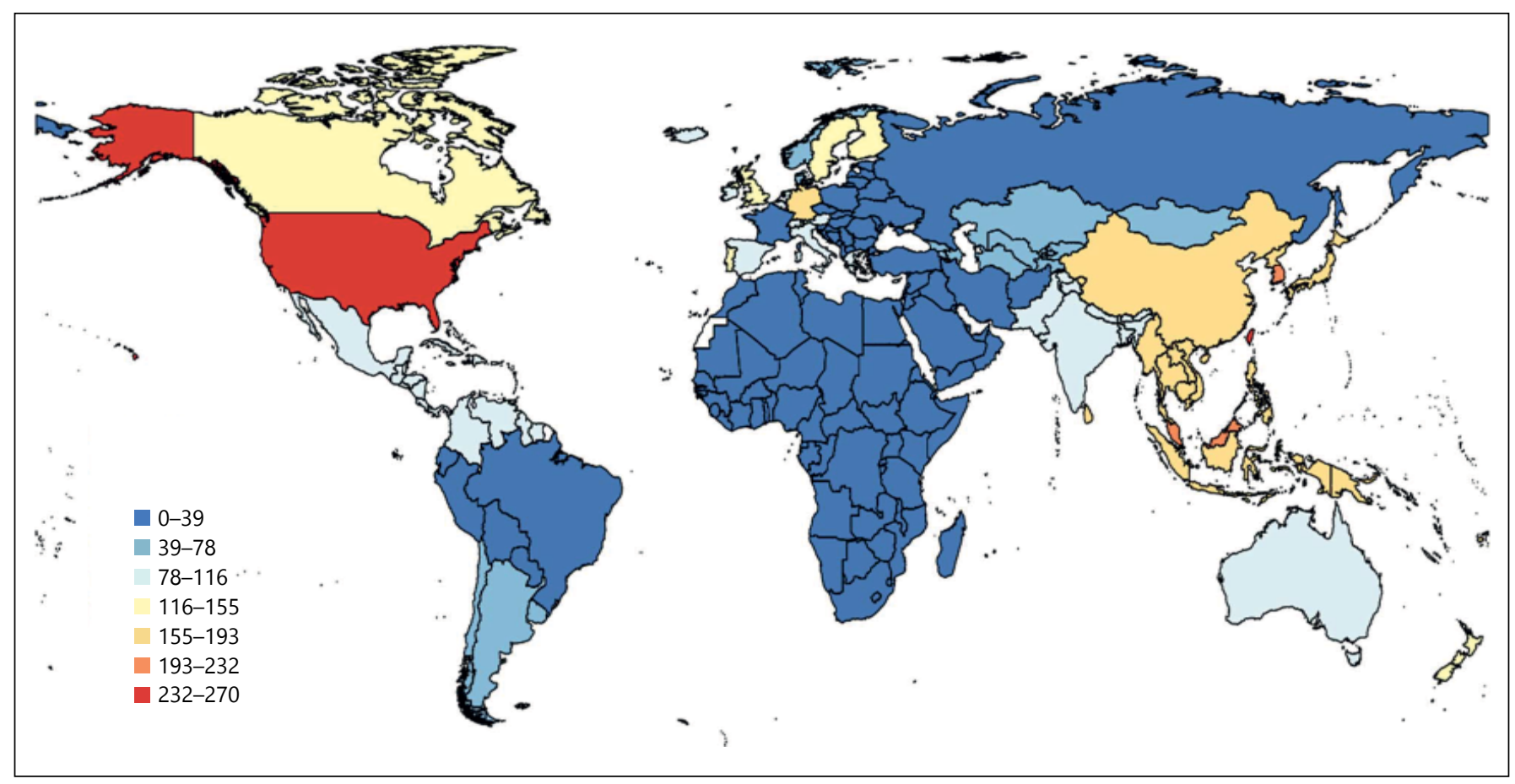

Fig. 2. Age-standardized annual prevalence (per 100,000) of hemorrhagic stroke in 2013. 


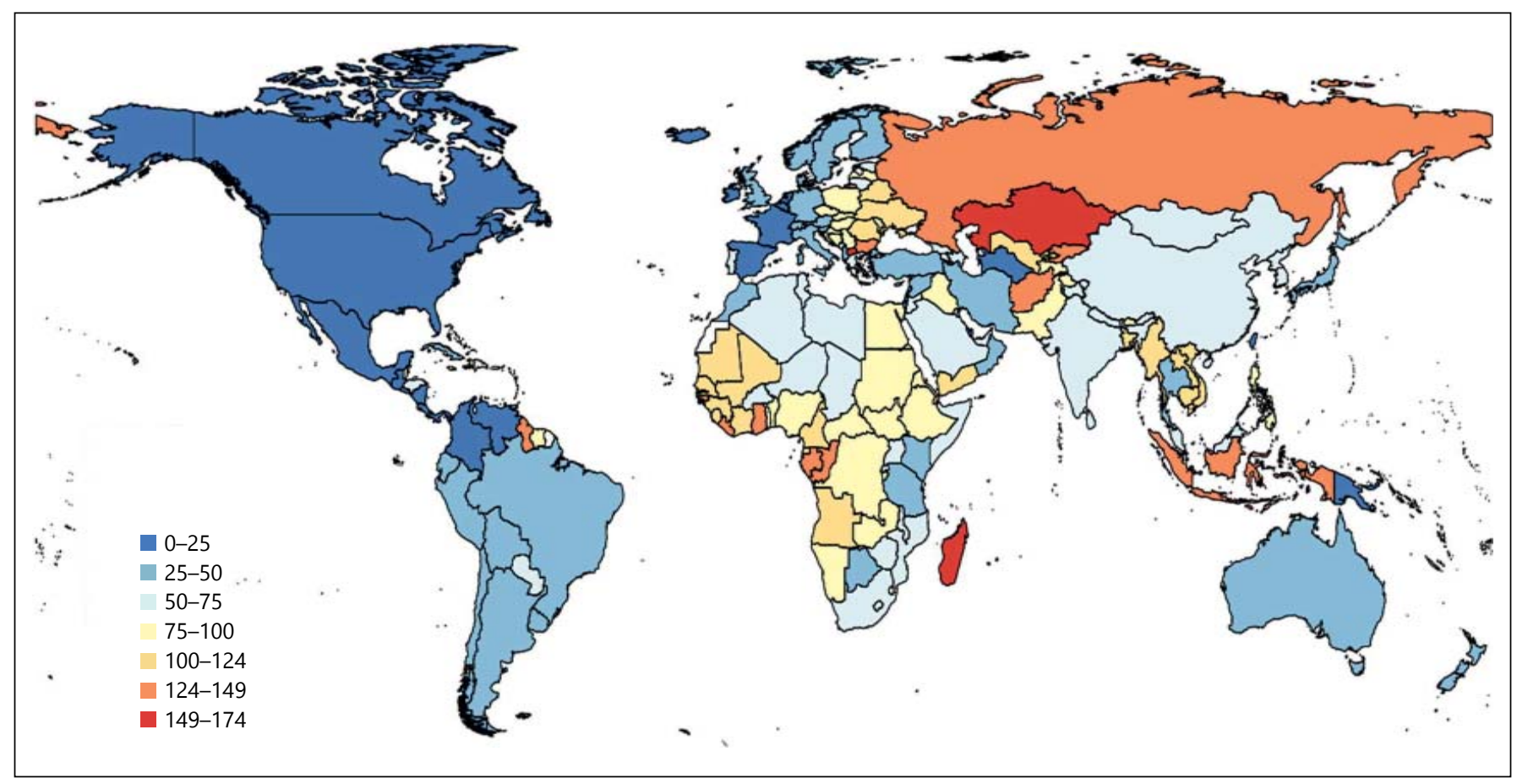

Fig. 3. Age-standardized annual mortality rates (per 100,000) of ischemic stroke in 2013.

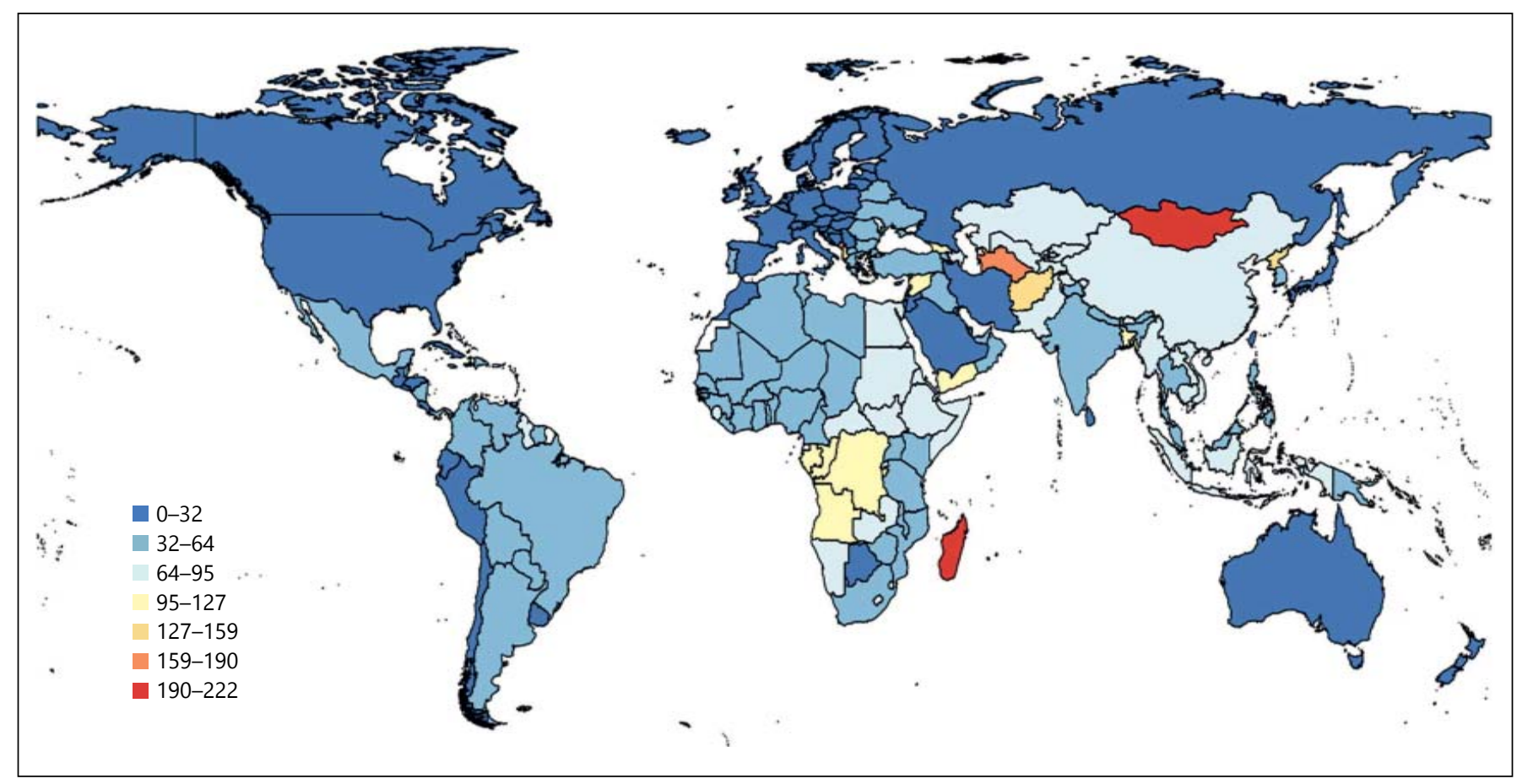

Fig. 4. Age-standardized annual mortality rates (per 100,000) of hemorrhagic stroke in 2013. 


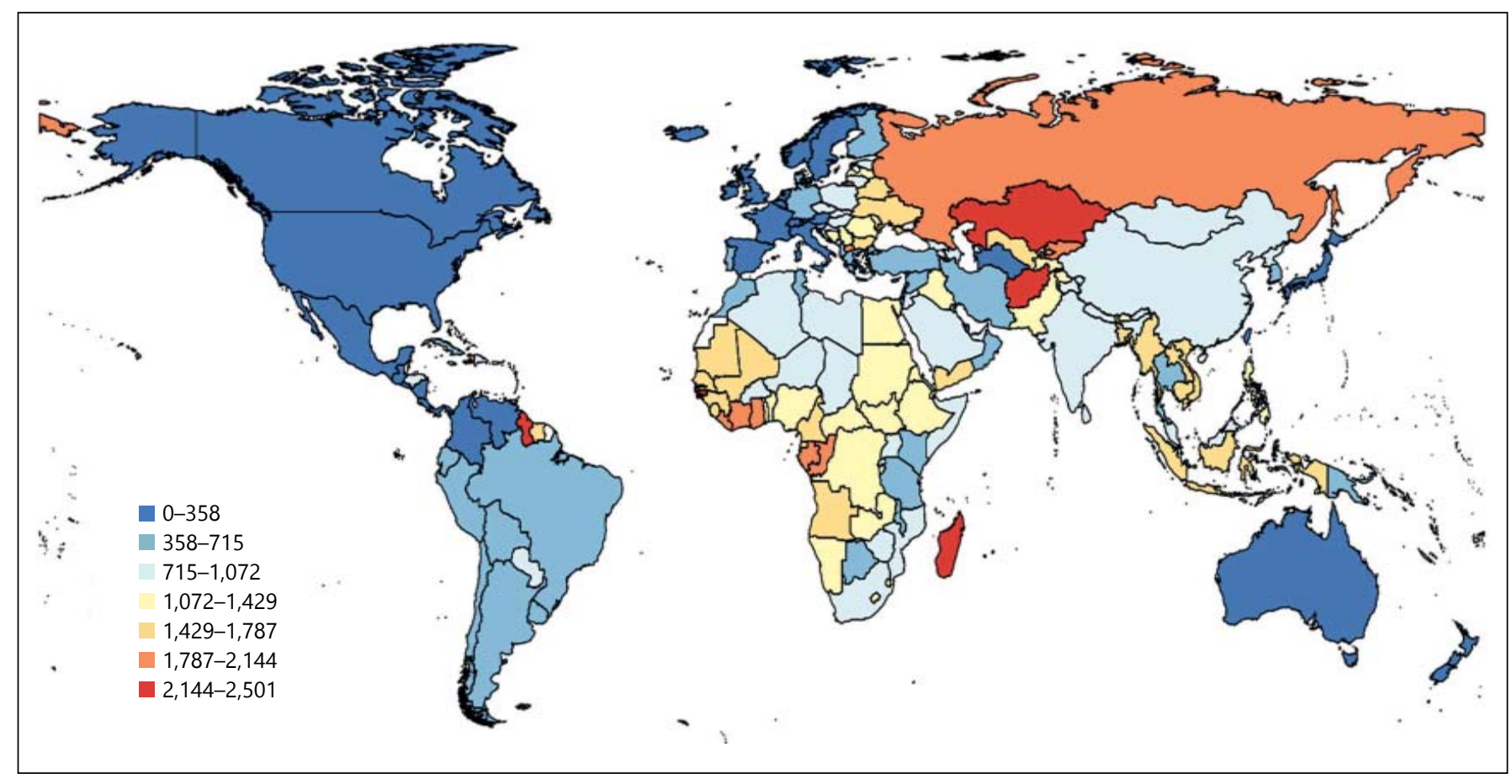

Fig. 5. Age-standardized annual DALYs rates (per 100,000) due to ischemic stroke in 2013.

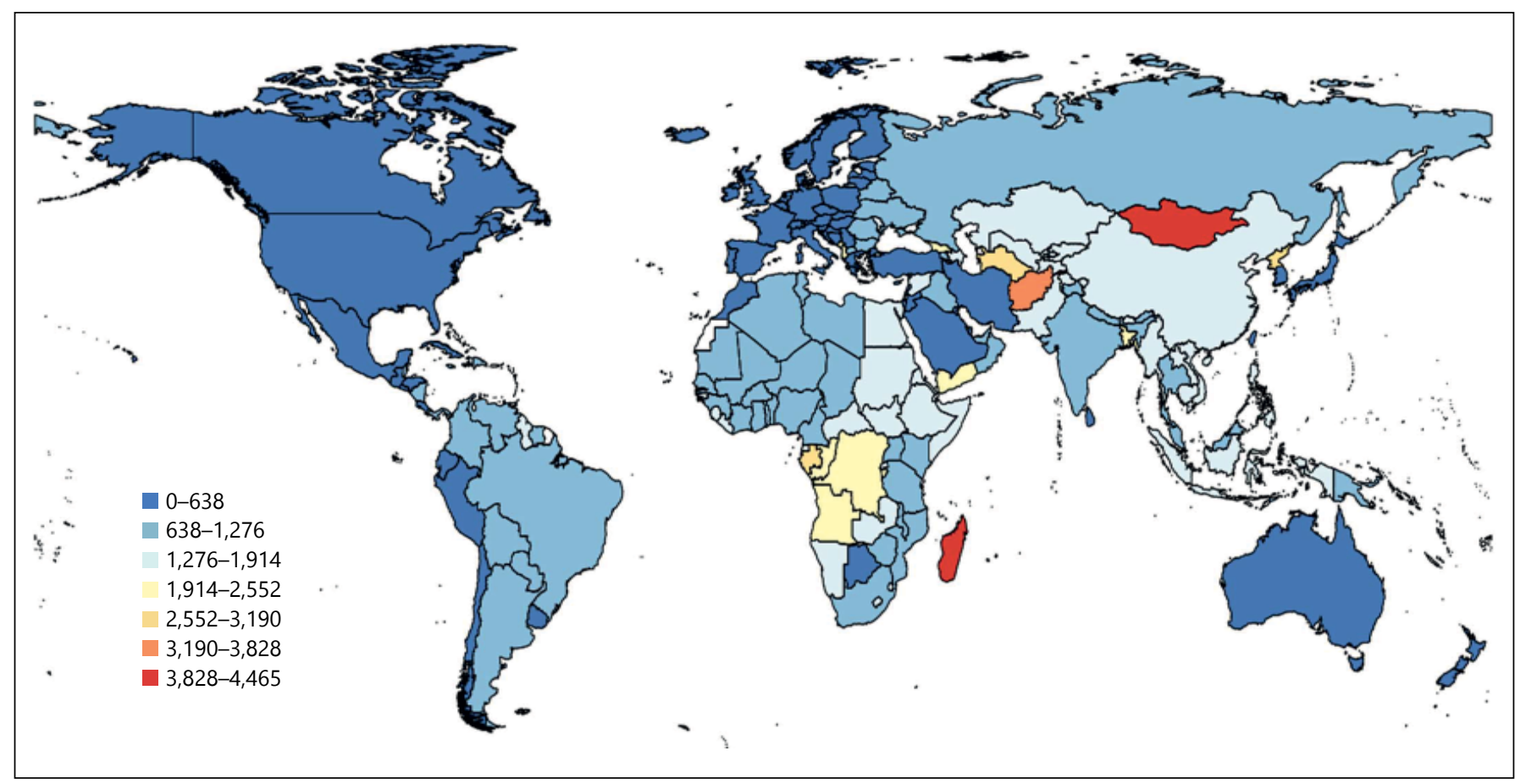

Fig. 6. Age-standardized annual DALYs rates (per 100,000) due to hemorrhagic stroke in 2013. 


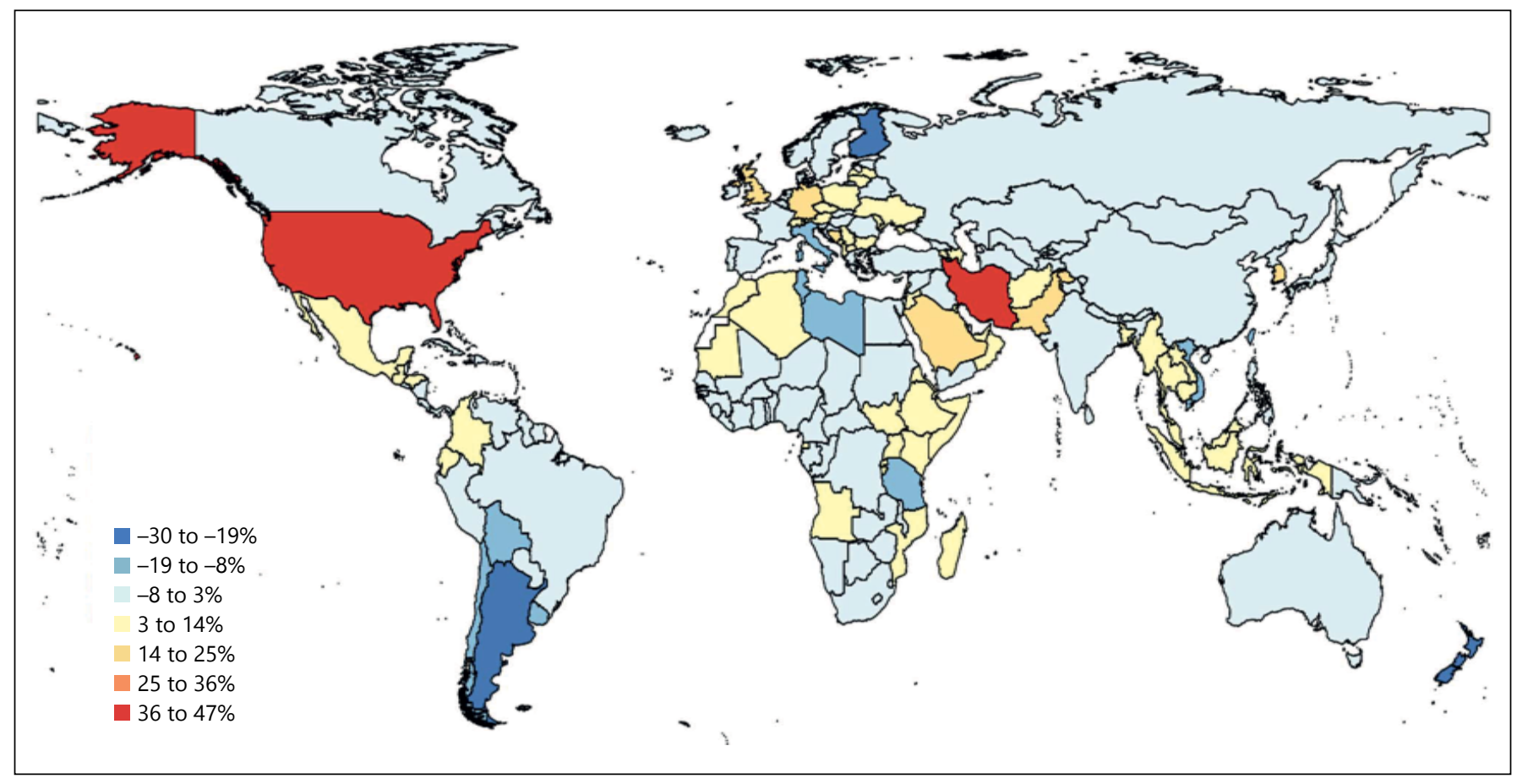

Fig. 7. Percent change in age-standardized prevalence due to ischemic stroke for 1990-2013.

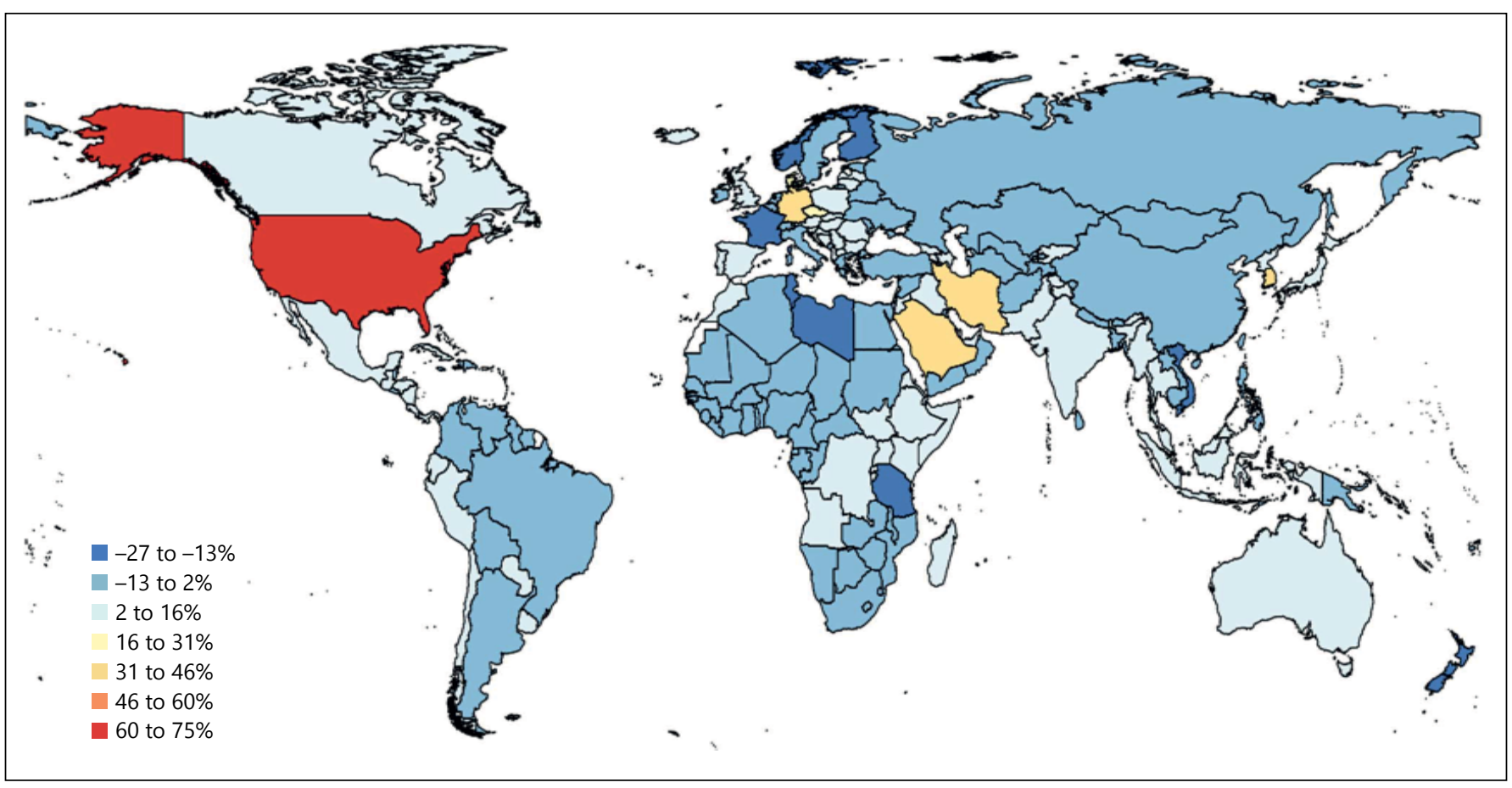

Fig. 8. Percent change in age-standardized prevalence due to hemorrhagic stroke for 1990-2013. 


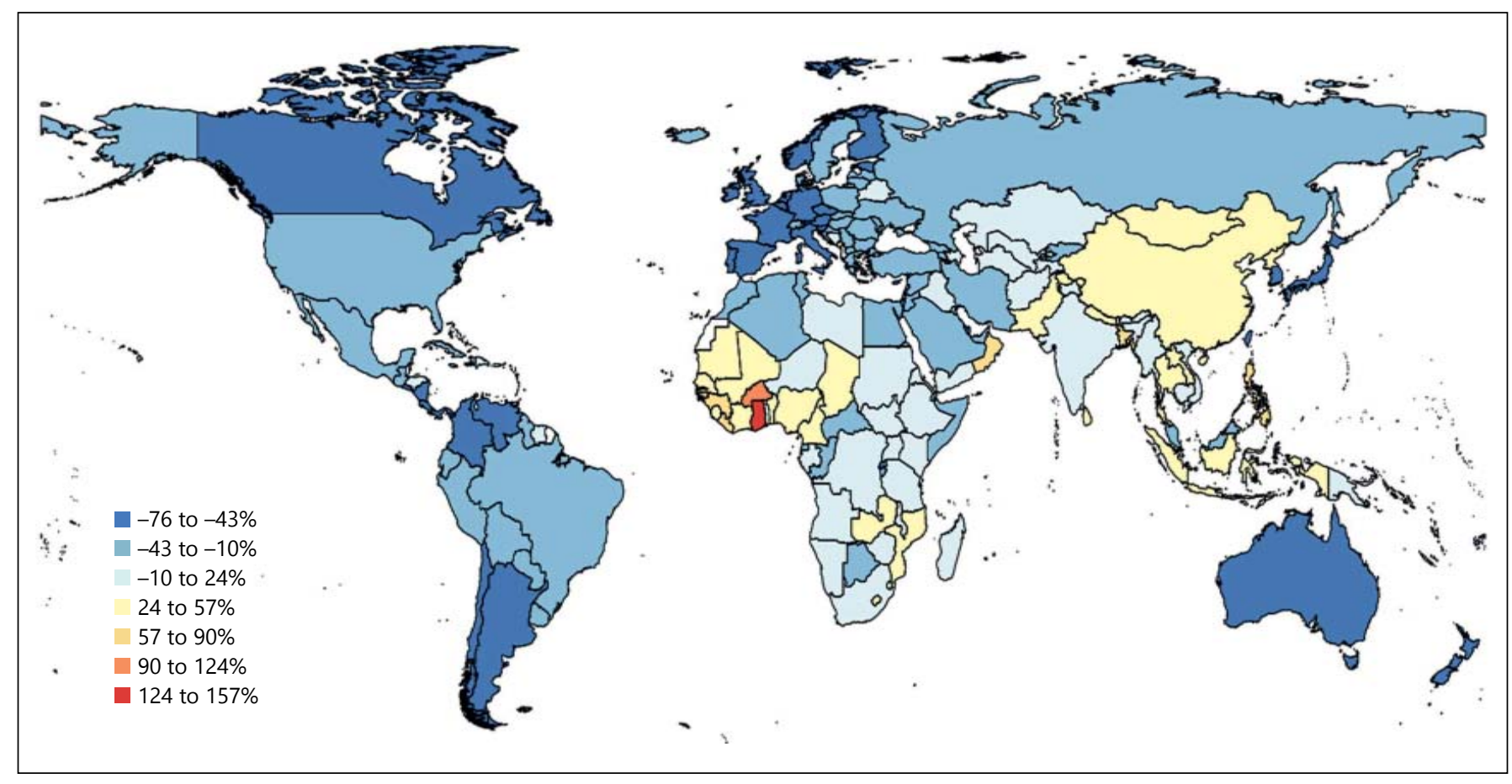

Fig. 9. Percent change in age-standardized mortality rates due to ischemic stroke for 1990-2013.

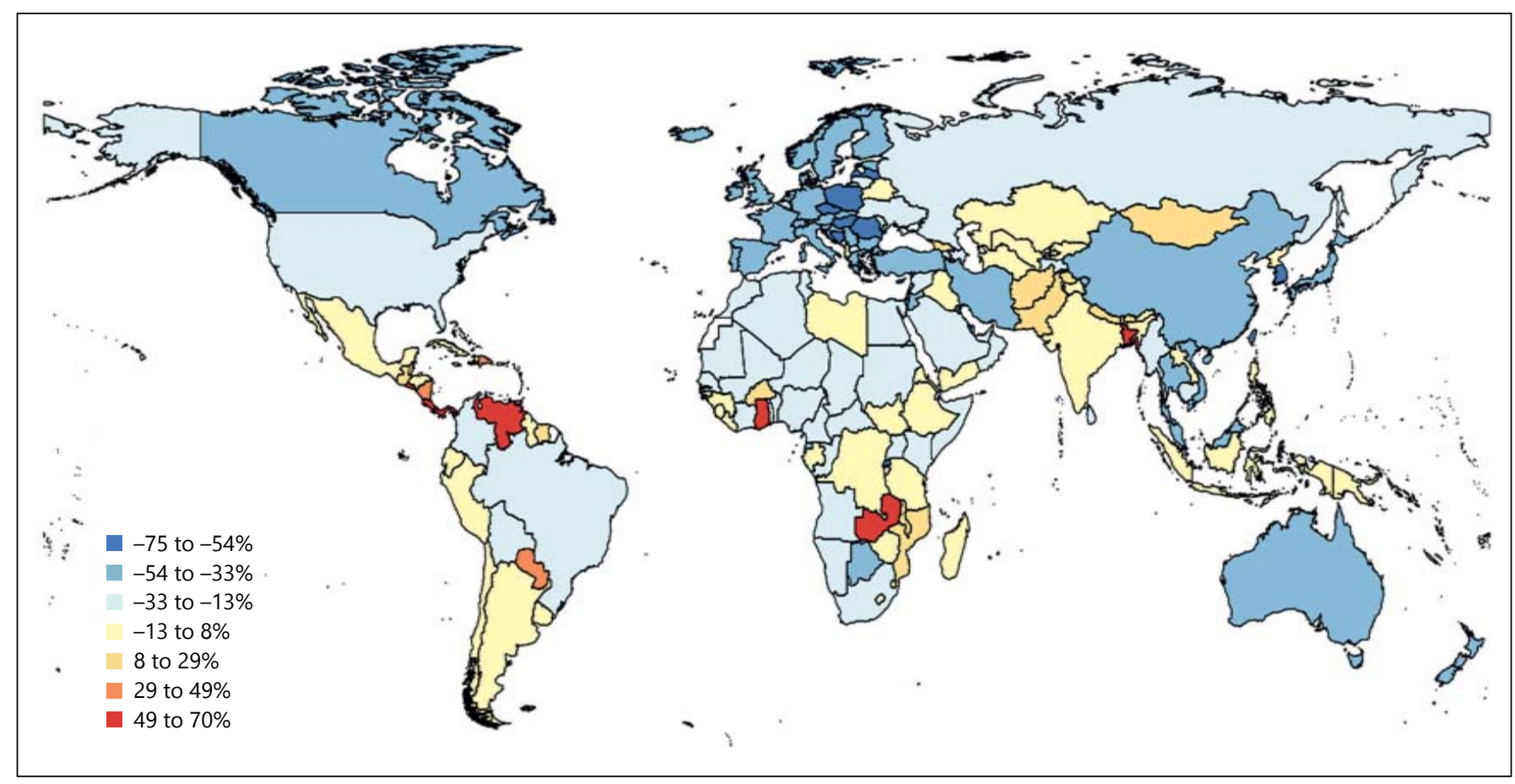

Fig. 10. Percent change in age-standardized mortality rates due to hemorrhagic stroke for 1990-2013. 


\section{Acknowledgments}

This work was undertaken as a part of the Global Burden of Diseases, Injuries, and Risk Factors (GBD 2013) study. We thank Sarah Safranek, University of Washington Health Sciences Library, for her help in developing the systematic review literature search strategies; Michael F. MacIntyre, Brittany Wurtz and Summer Ohno, University of Washington Institute for Health Metrics and Evaluation, for research coordination; Ryan M. Barber, University of Washington Institute for Health Metrics and Evaluation, for creating figures and tables for the paper; Hannah Gardener, University of Miami, for helping reviewing some studies; and Helen McDonald, AUT University National Institute for Stroke And Applied Neurosciences, for organizing teleconferences and secretarial support. The authors alone are responsible for the views expressed in this publication.

\section{Role of the Funding Source}

This study was funded by the Bill and Melinda Gates Foundation. The sponsor of the study had no role in the study design, data collection, data analysis, data interpretation or writing of the report. The GBD 2013 Core investigators had access to all data sources and were responsible for the content of the report and the decision to submit for publication.

\section{Disclaimer}

The views expressed in this article are those of the authors and do not necessarily represent the views of the National Heart, Lung, and Blood Institute; National Institutes of Health, or the US Department of Health and Human Services.

\section{Disclosure Statement}

The authors have no conflict of interest to declare.

\section{Appendix}

GBD 2013 Stroke Panel Experts Group (in Alphabetical Order by Country)

Argentina (Maria Cecilia Bahit); Australia (Amanda G. Thrift, Atte Meretoja, Bill Stavreski, Craig S. Anderson, Edwin Pearse, Geoffrey Donnan, Graeme J. Hankey, Mark T. Mackay, Stephen Davis, Zanfina Ademi); Austria (Michael Brainin); Azerbaijan
(Tural Guliyev); Bahrain (Randah R. Hamadeh); Barbados (Heather Harewood, Karen Springer); Brazil (Iuri da Costa Leite, Jefferson Gomes Fernandes, Norberto Luiz Cabral, Paulo A. Lotufo); Bulgaria (Klara Dokova); Canada (Farshad Pourmalek, Gabrielle DeVeber, Luciano A. Sposato, M. Patrice Lindsay, Patricia M. Riccio); Chile (Pablo M. Lavados); China (Bin Li, Chuanhua Yu, Guohong Jiang, Jixiang Ma, Maigeng Zhou, Ming Liu, Shankuan Zhu, Wenzhi Wang, Xiaofeng Liang, Yong Zhang); Colombia (Gabriel Alcalá-Cerra); Denmark (Hanne K. Christensen, Thomas Truelsen); Egypt (Foad Abd-Allah); Ethiopia (Awoke Temesgen, Berhe Weldearegawi Sahle, Semaw Ferede Abera, Yohannes Adama Melaku); Fiji (Devina Nand); France (Maurice Giroud); Germany (Jost B. Jonas, Matthias Endres, Ronny Westerman); Greece (Konstantinos Stroumpoulis); India (Dorairaj Prabhakaran, Jeyaraj Durai Pandian, Man Mohan Mehndiratta, Nobhojit Roy, Panniyammakal Jeemon, Rajeev Gupta, Vasanthan Rajagopalan); Indonesia (Soewarta Kosen, Tati Suryati Warouw); Iran (Reza Malekzadeh); Ireland (Martin J. O’Donnell); Israel (Natan M. Bornstein, David Tanne); Italy (Stefano Ricci, Valeria Caso); Japan (Yoshihiro Kokubo, Yukito Shinohara); Jordan (Majed Masoud Asad); Kenya (Vitalis Kizito Bwire); Korea (Sun Ha Jee, Young-Ho Khang); Mexico (Ismael Campos-Nonato); Malaysia (Kim Yunjin, Ramesh Sahathevan); Morocco (Fortuné Gankpé); Myanmar (Chaw Yin Myint); Netherlands (Johanna M. Geleijnse); New Zealand (Priya Parmar, Rita V. Krishnamurthi, Suzanne Barker-Collo, Valery L. Feigin); Nigeria (Rufus Olusola Akinyemi); Norway (Ole Norheim); Qatar (Shams Eldin Khalifa); Russia (Michael Kravchenko, Michael Piradov, Nikolay Shalamov, Vasiliy Victorovich Vlassov, Yuri Varakin); Rwanda (Jean De Dieu Ngirabega, Jean Pierre Nyemazi, Marie Aimee Muhimpundu); Saudi Arabia (Mohammad Saeedi, Neeraj Bedi); Singapore (Narayanaswamy Venketasubramanian); South Africa (Andre Pascal Kengne); Spain (David Rojas-Rueda, Ferrán Catalá-López); Sri Lanka (Samath D. Dharmaratne); Sweden (Bo Norrving, Rasmus Havmoeller); Uganda (Leo Atwine); United Kingdom (Amitava Banerjee, Charles Wolfe, Derrick A. Bennett, Finbar O'Callaghan, Ivy Shiue, Julia A. Critchley, Majid Ezzati, Michael Soljak, Myles D. Connor, Peter M. Rothwell, Rajiv Chowdhury, Rustam Al-Shahi Salman, William Whiteley, Zhengming Chen); Uruguay (Mercedes Colomar); USA (Adnan M. Durrani, Anand Dayama, Andrew E. Moran, Awoke Misganaw, Brett M. Kissela, Catherine Amlie-Lefond, Catherine O. Johnson, Cheng Huang, Christopher J.L. Murray, Chugh Sumeet, Daniel Kim, David K. Cundiff, David Lawrence Tirschwell, Dhruv S. Kazi, Dima Qato, Edmond Kato Kabagambe, Eric Ding, Gene Bukhman, Gene Kwan, George A. Mensah, George D. Thurston, Grant Nguyen, Gregory A. Roth, Josef Coresh, Kate Lefondulq, Kevin N. Sheth, Matthew A. Corriere, Mohammad H. Forouzanfar, Mohsen Naghavi, Nana Mainoo, Norman J. Beauchamp, Ralph L. Sacco, Richard F. Gillum, Sanjay Basu, Stephen M. Schwartz, Sumeet Chugh, Teresa Fung, Theo Vos, Tim E. Byers, Uchechukwu K.A. Sampson, Walter A. Rocca, Warren Lo). 